\section{Overfishing in west Africa by EU vessels}

China is not the only culprit threatening the marine ecosystems of west Africa through overfishing (Nature $496,18 ; 2013)$. The region has been the fish basket of southern Europe since the 1960s, and the European Union (EU) is going to great lengths to renew its fishery agreements with African nations.

These agreements are questionable because they promote the export of African marine resources into the EU to the detriment of local economies and food sovereignty. Morocco has allowed EU vessels into the national waters of neighbouring Western Sahara, effectively hijacking its fish stocks.

The uncontrolled harvesting of pelagic fish and octopus by hundreds of foreign fishing vessels is having a deep ecological impact on the Atlantic Ocean's Canary Current upwelling marine ecosystem, a major biodiversity hotspot. It is also destroying vast amounts of by-catch.

Research programmes are under way to quantify this collateral damage. Some highly vulnerable and endangered marine vertebrates are affected, including monk seals, several shark species, dolphins and sea turtles, and hundreds of thousands of Macaronesian and Palaearctic seabirds that breed and overwinter along the west African coast (see, for example, J. Zeeberg et al. Fish. Research 78, 186-195; 2006).

We therefore urge EU parliament members to rethink their African fishing policies, properly taking into account the devastating political, social and ecological impact of their large industrial fisheries.

Rauil Ramos Centre for Functional and Evolutionary Ecology (CEFE), CNRS, UMR5175, Montpellier, France. raul.ramos@cefe.cnrs.fr David Grémillet CEFE-CNRS Montpellier, France; and PFIAO, DST/NRF Centre of Excellence,
University of Cape Town, South Africa.

\section{Scale of global road map is impractical}

William Laurance and Andrew Balmford's proposed global zoning map (Nature 495, 308-309; 2013) for road planning would be too crude in scale to be useful for practical, on-theground decisions.

Positive outcomes for biodiversity from road building are more likely to be achieved in a local context. Decisions about road developments are not made on a global scale, and rarely even on a national level.

Any road planned with environmental and social considerations in mind is better than one designed without them. But decision-makers should not shift their focus too far from the major problems that proposed roads might create.

Malgorzata Blicharska Swedish University of Agricultural Sciences, Uppsala, Sweden. malgorzata.blicharska@slu.se

\section{Uphold standards for lab reagents}

Scientists assume that reagents supplied commercially have been rigorously tested for purity. We have found that this assumption can be risky.

Our laboratory purchased 3 milligrams of a biomolecule from a reputable supplier to investigate its effect on nerve cells. Anomalous results forced us to test its purity. Using mass spectrometry and qualitative western blotting, we discovered unacceptable levels of contamination with several other biologically active molecules.

Several research groups have published important mechanistic conclusions based on the activity of this molecule. Contamination with other active components would call these results into question.
Reagents that are misleadingly labelled waste researchers' funds and time. Suppliers should declare the accurate composition and modes of analysis for every batch of their products.

Sally Roberts, Heidi Fuller Keele University, Staffordshire, UK; and Robert Jones \& Agnes Hunt Orthopaedic Hospital, Oswestry, UK. sally.roberts@rjah.nhs.uk Bruce Caterson Cardiff University, UK.

\section{Crowd-sourced soil data for Europe}

Since its launch by the UK Natural Environment Research Council in June 2012, the free iPhone app mySoil has attracted more than 2.6 million web hits and 12,500 dedicated users (see go.nature.com/tylukf). Next month, a new version will extend its coverage to soils in the European Union (EU).

Users of mySoil can currently view soil maps of the United Kingdom (excluding Northern Ireland) that provide regional information on soil depth, texture, $\mathrm{pH}$ and organic-matter content, and on vegetation habitats. They can upload photos and descriptions of their local soils. More than 500 entries have so far contributed to this valuable data bank of soil properties in different localities.

The remarkable success of collecting data in this way reflects widespread public interest and promotes communication with the science community.

With infrastructure covering an estimated average of $9 \%$ of the EU - often spread across the most productive soils - and with sustainable land management becoming increasingly urgent, mySoil's democratization of soil data is a timely development. Wayne Shelley, Russell Lawley British Geological Survey, Nottingham, UK. David A. Robinson Centre for Ecology \& Hydrology, Bangor, UK. davi2@ceh.ac.uk

\section{Chinese institute clarifies patent case}

As director and deputy director of the Institute of Microbiology, Chinese Academy of Sciences (IMCAS), we would like to clarify some points in your discussion of our involvement in an intellectual-property case (Nature 492, 323; 2012).

Without our knowledge, Shandong Hilead Biotechnology in Laiyang - a recipient of the dodecanedioic acid technology invented by IMCAS, with which Hilead collaborated to further improve this technology - filed a patent application in 2010 that listed both of us and five others as inventors (patent CN201010160266.4).

When we discovered this, we demanded that our names be dropped from the application because we had not made any technical contribution to the patent. Hilead filed an application for a change of inventors listed on the patent on 25 January 2011 to the State Intellectual Property Office of China, who approved the change on 14 February 2011 (file 2011021000026950). So the removal of our names occurred a few months before two employees at Cathay Industrial Biotech in Shanghai filed a lawsuit over the patent in June 2011.

Hilead officially apologized on 17 August 2011 in a letter to IMCAS for using our names without consent. The court decision on the lawsuit exempted us from civil liability and no apology was required from us (Beijing Higher People's Court decision 3975, 16 November 2012). Li Huang, Shuang-Jiang Liu IMCAS, Beijing, China. huangl@im.ac.cn

\section{CORRECTION}

A declaration of competing financial interests was omitted from the Comment 'A jumpstart for electroceuticals'

(Nature 496, 159-161; 2013).

This has been added online. 